\title{
Suppression of PTEN/AKT signaling decreases the expression of TUBB3 and TOP2A with subsequent inhibition of cell growth and induction of apoptosis in human breast cancer MCF-7 cells via ATP and caspase-3 signaling pathways
}

\author{
ZHENHUA YANG $^{1 *}$, YING LIU ${ }^{1 *}$, CHANGZHENG SHI $^{2}$, YUQIN ZHANG ${ }^{1}$, RONGZHAO LV $^{3}$, \\ RONG ZHANG $^{1}$, QIAN WANG ${ }^{1}$ and YIMING WANG ${ }^{1}$ \\ ${ }^{1}$ Department of Oncology, ${ }^{2}$ Medical Imaging Center, and ${ }^{3}$ Department of Surgery on Galactophore, \\ The First Affiliated Hospital of Jinan University, Guangzhou 510630, P.R. China
}

Received May 30, 2016; Accepted July 8, 2016

DOI: $10.3892 / o r .2017 .5358$

\begin{abstract}
The aim of the present study was to evaluate the effects of PTEN/AKT signaling on TUBB3 and TOP2A expression and on the subsequent cell growth of human breast cancer MCF-7 cells. We found that the disease-free survival (DFS) and overall survival (OS) of breast cancer patients with TUBB3-positive tumors were lower than these rates in the patients with TUBB3-negative tumors. Meanwhile, DFS and OS of breast cancer patients with TOP2A-positive tumors were also lower than these rates in patients with TOP2A-negative tumors. Suppression of PTEN reduced the protein expression of TUBB3 and TOP2A in MCF-7 cells. Suppression of PTEN also reduced cell proliferation and induced apoptosis and caspase-3 activity in MCF-7 cells. Moreover, an increase in ATP also reduced TUBB3 and TOP2A protein expression, reduced cell proliferation and induced apoptosis and caspase-3 activity in the MCF-7 cells following suppression of PTEN. Suppression of phosphorylation-AKT (p-AKT) reduced the protein expression of TUBB3 and TOP2A in the MCF-7 cells. Suppression of p-AKT also reduced cell proliferation and induced apoptosis and caspase-3 activity in the MCF-7 cells. Then, ATP also reduced TUBB3 and TOP2A protein expression, reduced cell proliferation and induced apoptosis and caspase-3 activity in MCF-7 cells following suppression of p-AKT. These results suggest that PTEN/AKT signaling affects the expression of TUBB 3 and TOP2A reducing cell growth and inducing apoptosis of human breast cancer MCF-7 cells through ATP and caspase-3 signaling pathways. TUBB3
\end{abstract}

Correspondence to: Dr Yiming Wang, Department of Oncology, The First Affiliated Hospital of Jinan University, 613 Huangpu Road West, Guangzhou 510630, P.R. China

E-mail:wangymgz@163.com

${ }^{*}$ Contributed equally

Key words: PTEN, AKT, TUBB3, TOP2A, breast cancer and TOP2A may be promising prognostic markers for the efficacy of adjuvant cisplatin-based chemotherapy.

\section{Introduction}

Breast cancer is one of the most common female malignant tumors worldwide. According to the statistics from the International Agency for Research on Cancer (IARC), $\sim 1.4$ million women suffer from breast cancer, and $\sim 500,000$ individuals die of breast cancer every year (1). The latest statistics released by Cancer Journal for Clinicians indicated that 230,000 women in the US were diagnosed with breast cancer in 2011 , accounting for $30 \%$ of all newly diagnosed female malignant tumors, with an incidence rate ranked as no. 1 among all female malignant tumors (2). Although China is a region with a relatively low incidence of breast cancer, in recent years, the incidence shows an increasing trend (3). Particularly in Beijing, Shanghai and other big cities, the incidence rate has risen to 50-60/100,000 individuals, achieving the highest incidence rate of all female cancers (4). In the past 20 years, with the development and application of chemotherapy, hormonal and targeted therapies, and other combined modality therapeutic methods, breast cancer patients have a significantly prolonged lifespan, and the 5-year survival rate increased from 55\% during the 1970 s to the present rate of $80 \%$. In some countries, the breast cancer mortality rate has shown a downward trend (5).

In recent years, researchers have given more attention to the relationship between molecular markers and chemotherapeutic drug resistance, and have achieved some results (6). Platinum agents are a group structurally similar to bifunctional alkylating agents, which forms a complex with bases on nucleophilic groups by covalent bonding, resulting in DNA replication and transcription by structural changes and disorders, thereby inhibiting cell division (7). Numerous studies have mainly focused on the expression of TUBB3 and taxane resistance (8). Research has shown that TUBB3 is not only a major component of tubulin, with important significance for the maintenance of cell shape, but its expression in tumor 
tissues is also related to the sensitivity of chemotherapeutic agents (6). Research has shown that TUBB3 expression is closely related to drug resistance of taxanes. TUBB3 can reduce the gathering speed of tubulin and resist against the tubulin aggregation caused by taxane chemotherapy drugs, leading to a decrease in the sensitivity to chemotherapy drugs, thus causing the failure of chemotherapy (9).

In the 1970s, it was found that the main mechanism of anthracycline is to inhibit TOP2A, leading to the breakage of double-stranded DNA, thereby promoting tumor cell apoptosis by interacting with the TOP2A-DNA complex (10). TOP is a key ribozyme necessary for DNA replication and transcription processes, which can be classified into TOP1 and TOP2 according to the nature. TOP1 causes the breakage of single-stranded DNA on DNA sugar-phosphate backbone, while TOP2 mainly causes the breakage of double-stranded DNA (11). In eukaryotic organisms, TOP2 can be classified into two types of isoenzymes, TOP2A and TOP2B. The TOP2A gene is adjacent to the HER-2 gene, located in the 17q21-q22 region (as the encoding gene of DNATC) P20t. The gene product is a homodimer protein with a relative molecular mass of 170,000, involved in the processes of DNA recombination, repair, replication and transcription (12). The TOP2A protein level has a significant cycle-specificity in proliferative cells, of which the expression is low in the G1 phase, increased in the $\mathrm{S}$ phase, and reaches a peak after the G2-M phase. The overexpression of TOP2A may reflect cell proliferation activity and aggressive invasive behaviors and poor prognosis (10). Therefore, TOP2A plays an important role in the study of cytobiology.

As an important tumor-suppressor gene following P53 and $\mathrm{PRb}$, the PTEN gene is closely related to the development of various human malignant tumors, biological behavior and prognosis. The PTEN gene is located in the 10q23.3 region that has high deletion incidence of human chromosomes and heterozygotes, and is the first tumor-suppressor gene shown to have phosphatase activity to date (13). Researchers have found abnormalities in mutation, deletion and protein expression, thus losing the negative regulatory effect on cell growth, which may eventually lead to tumorigenesis (14). In the normal human, the PTEN expression level is high in placenta, heart, brain, lung and kidney and other tissues, and it is also expressed in peripheral blood mononuclear cells. Meanwhile PTEN is a self-renewal regulatory gene for normal hematopoietic stem cells and a leukemia promoter gene, which is also essential for the maintenance of normal hematopoiesis. Mutations, deletion and decreased expression of PTEN are found in leukemia, lymphoma and multiple myeloma and other blood and lymphoid malignancies, highly suggestive of the important molecular regulatory function of PTEN in hematopoietic cell proliferation, apoptosis and malignant transformation (14). In the present study we examined the PTEN/AKT signaling effects on TUBB3 and TOP2A expression and cell growth of human breast cancer MCF-7 cells.

\section{Materials and methods}

Patients and treatment. A total of 57 breast cancer patients were recruited for the present study from the Department of Oncology, The First Affiliated Hospital of Jinan University,
Table I. Primer sequences used in the present study.

\begin{tabular}{lll}
\hline Gene name & \multicolumn{2}{c}{ Primer sequences } \\
\hline TUBB3 & F & 5'-ACTACAACGAGGCCTCTTCTCAC-3' \\
& R & 5'-TTGTTGCCGGCCCCACTCTGACC-3' \\
TOP2A & F & 5'-ATCCTGCCAAAACCAAGAATCG-3' \\
& R & 5'-GTACAGATTTTGCCCGAGGAGC-3' \\
GAPDH & F & 5'-GTGAACCATGAGAAGTATGACAA-3' \\
& R & 5'-CATGAGTCCTTCCACGATAC-3'
\end{tabular}

F, forward; R, reverse.

between February 2010 and October 2010. After surgery, all patients were surveyed for 2 months. All participants provided written informed consent and the present study was approved by the Ethics Committee of The First Affiliated Hospital of Jinan University.

Immunohistochemical. Breast cancer and para-carcinoma tissues were fixed with $10 \%$ formalin for $24 \mathrm{~h}$ and embedded in paraffin. Tissues were sliced into $4-\mu \mathrm{m}$ sections and baked for $1 \mathrm{~h}$ at $65^{\circ} \mathrm{C}$. The sections were exposed to $10 \mathrm{mM}$ citrate buffer (Beyotime, Shanghai, China) for $10 \mathrm{~min}$ at $37^{\circ} \mathrm{C}$ and incubated with the mouse monoclonal anti-human-TUBB3 and anti-human-TOP2A (both from Sigma-Aldrich, St. Louis, MO, USA). The tissue sections were incubated with polyclonal goat anti-rabbit IgG biotinylated secondary antibodies at $37^{\circ} \mathrm{C}$ for $1 \mathrm{~h}$. Then, the sections were incubated with streptavidin-horseradish peroxidase complex (Sigma-Aldrich) at $37^{\circ} \mathrm{C}$ for $10 \mathrm{~min}$. The sections were evaluated independently under a light microscope (CX21; Olympus Corporation, Tokyo, Japan).

qPCR analysis. Total RNA was isolated from tissue samples using TRIzol reagent (Takara, Shiga, Japan). Total RNA $(1-2 \mu \mathrm{g})$ was reverse transcribed into cDNA using a reverse transcription kit (Takara). The expression of TUBB3 and TOP2A was measured using SYBR-Green I Master Mix (Takara) and qPCR (7500 ABI System; Applied Biosystems, Foster City, CA, USA). The qPCR reaction was performed as follows: $95^{\circ} \mathrm{C}$ for $5 \mathrm{~min}$, followed by 40 cycles of denaturation at $95^{\circ} \mathrm{C}$ for $20 \mathrm{sec}$ and annealing/extension at $60^{\circ} \mathrm{C}$ for $30 \mathrm{sec}$. Primer sequences used in the study are listed in Table I.

Cell culture. Human breast cancer MCF-7 cells were cultured in RPMI-1640 medium supplemented with $10 \%$ fetal bovine serum (FBS) and antibiotic solution $(100 \mu \mathrm{g} / \mathrm{ml}$ streptomycin and $100 \mathrm{U} / \mathrm{ml}$ penicillin (all from HyClone, Logan, UT, USA) at $37^{\circ} \mathrm{C}$ in a $5 \% \mathrm{CO}_{2}$ incubator.

Cell viability assay. Cell viability was evaluated using the MTT assay. MCF-7 cells $\left(2 \times 10^{4} /\right.$ well $)$ in a 96 -well plate were treated with VO-OHpic trihydrate $(5 \mu \mathrm{M})$, a specific PTEN inhibitor; or 3-methyladenine (3-MA; $10 \mu \mathrm{M})$, a PI3K inhibitor; or ATP $(5 \mu \mathrm{M})$ for 0,24 and $48 \mathrm{~h}$. Ten milliliters $(5 \mathrm{mg} / \mathrm{ml})$ of MTT solution was added to each well for a 4-h period at $37^{\circ} \mathrm{C}$. Dimethyl sulfoxide (DMSO) was added into 

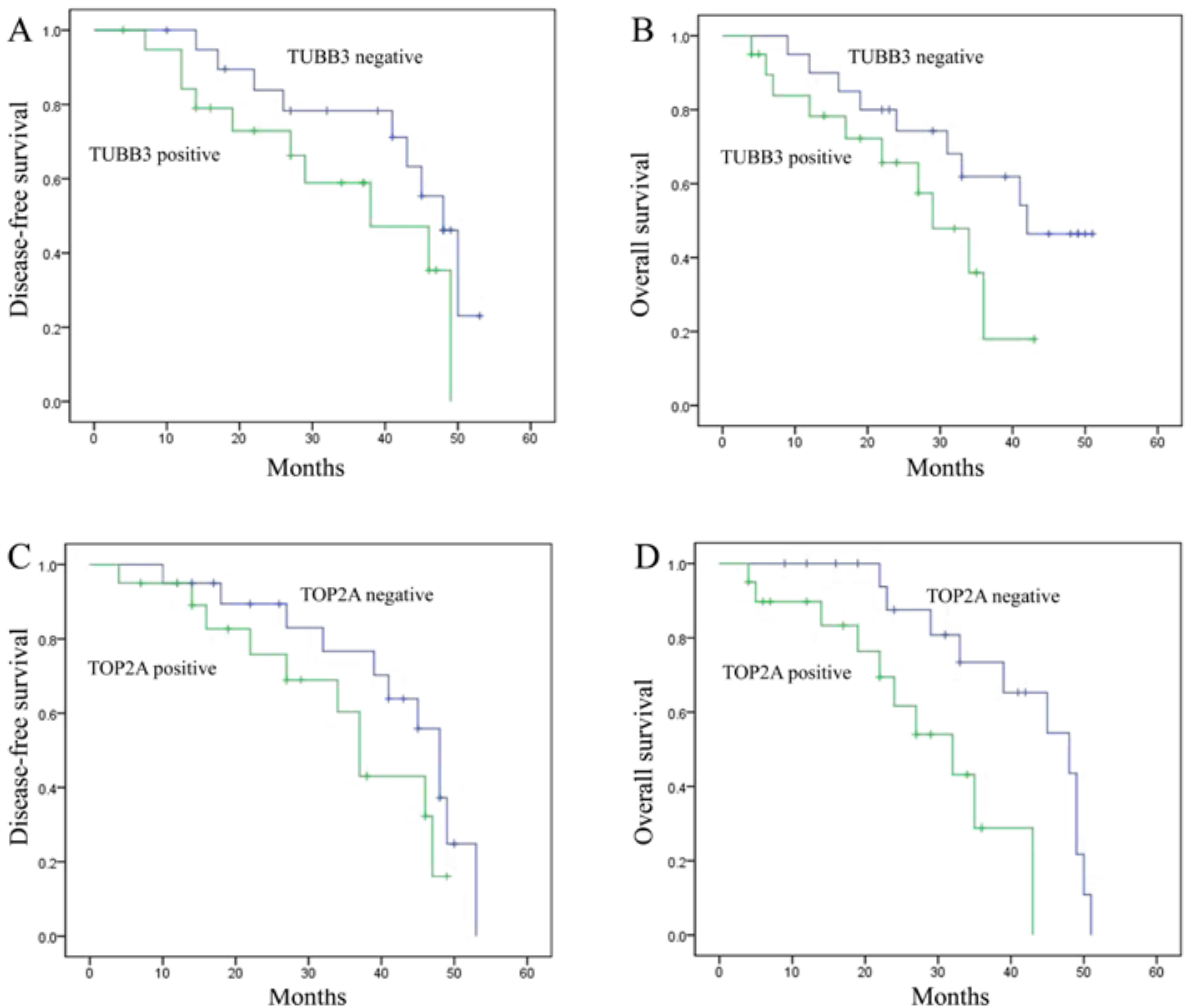

Figure 1. Disease-free survival (DFS) and overall survival (OS) of the breast cancer patients. (A) DFS and (B) overall survival of the breast cancer patients with TUBB3-positive and TUBB3-negative tumors. (C) DFS and (D) OS of the breast cancer patients with TOP2A-positive and TOP2A-negative tumors.

each well and dissolved for $30 \mathrm{~min}$. Absorbance (OD490) was assessed using a FLUOstar OPTIMA microplate reader (BMG Labtech, Cary, NC, USA) at $490 \mathrm{~nm}$.

Cell apoptosis analysis. MCF-7 cells $\left(1-2 \times 10^{6} /\right.$ well) in a 6 -well plate were treated with $\mathrm{VO}-\mathrm{OHpic}$ trihydrate $(5 \mu \mathrm{M})$ or 3-MA $(10 \mu \mathrm{M})$ for $48 \mathrm{~h}$. After collection and washing with phosphate-buffered saline (PBS), the cells were stained with Annexin V and propidium iodide (PI) using the Annexin V-PI detection kit (Roche, Mannheim, Germany). Cell apoptosis was measured using flow cytometric analysis using a FACScan (BD Biosciences, San Jose, CA, USA).

Caspase-3 activity assay. Cells were lysed using RIPA lysis buffer and the protein content of the cell lysate was quantified by the BCA protein assay (both from Beyotime). Caspase-3 activity was measured in $200 \mu \mathrm{g}$ of cell lysate using Ac-DEVD-pNA (Beyotime) for $2 \mathrm{~h}$ at $37^{\circ} \mathrm{C}$. Absorbance (OD490) was measured using a FLUOstar Optima microplate reader (BMG Labtech, Cary, NC, USA) at a wavelength of $405 \mathrm{~nm}$.

Western blot analysis. Cells were washed twice with PBS and were lysed in RIPA lysis buffer for $30 \mathrm{~min}$ at $4^{\circ} \mathrm{C}$. The concentration of total protein was measured uisng a Bradford kit (\#500-0205; Bio-Rad, Hercules, CA, USA). Equal amounts of protein $(50 \mu \mathrm{g})$ were boiled and 10-12\% SDS-PAGE was carried out. The proteins were transferred onto nitrocellulose membranes (Bio-Rad). Blots were blocked in 5\% skim milk in Tris-buffered saline Tween-20 (TBST) and incubated with anti-Bax (14796; 1:1,000), anti-TUBB3 (5568; 1:1,000),
anti-TOP2A (12286; 1:1,000), anti-p-AKT (4060; 1:1,000) (all from Cell Signaling Technology, Inc., Danvers, MA, USA) and GAPDH $\left(1: 2,000\right.$; Beyotime) at $4^{\circ} \mathrm{C}$ overnight. Blots were washed twice with TBST and incubated with secondary antibodies (6401-05; Amyjet Scientific, Inc., Wuhan, China) prior to identification of bands with chemiluminescence (ECL; Beyotime). The density was quantified using ImageJ software 3.0 (Bio-Rad).

Statistical analysis. All data are presented as mean values and standard deviation (mean $\pm \mathrm{SD}$ ). One-way ANOVA with Tukey's post hoc comparisons at $\mathrm{P}<0.05$ was considered to indicate a statistically significant result.

\section{Results}

Disease-free survival (DFS) or overall survival (OS) of the breast cancer patients. Firstly, we determined the DFS and OS of human breast cancer patients and analyzed the relationships of DFS and OS with expression of TUBB3 and TOP2A. As shown in Fig. 1A and B, the DFS and OS of the breast cancer patients with TUBB3-positive tumors were reduced when compared with the DFS and OS in patients with TUBB3negative tumors. Meanwhile, the DFS and OS of breast cancer patients with TOP2A-positive tumors were also reduced when compared with the DFS and OS in patients with TOP2Anegative tumors (Fig. 1C and D).

Expression of TUBB3 and TOP2A in the breast cancer patient tissue. Next, we found that the TUBB3 protein and miRNA expression in breast cancer tissues were observably higher 

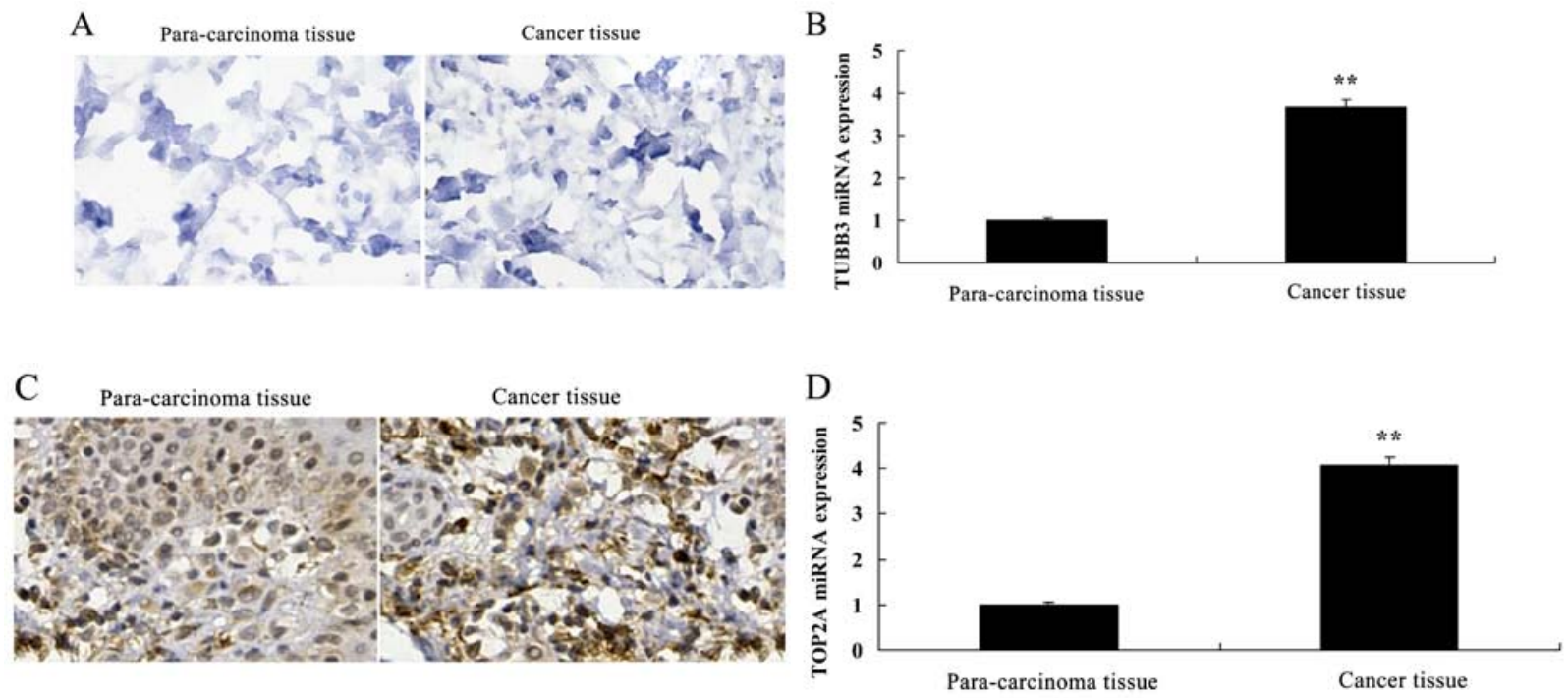

Figure 2. Expression of TUBB3 and TOP2A in breast cancer patient tissues. (A) TUBB3 protein and (B) miRNA expression in breast cancer patients. (C) TOP2A protein and (D) miRNA expression in breast cancer patients; ${ }^{* *} \mathrm{p}<0.01$ vs. the para-carcinoma tissue group.
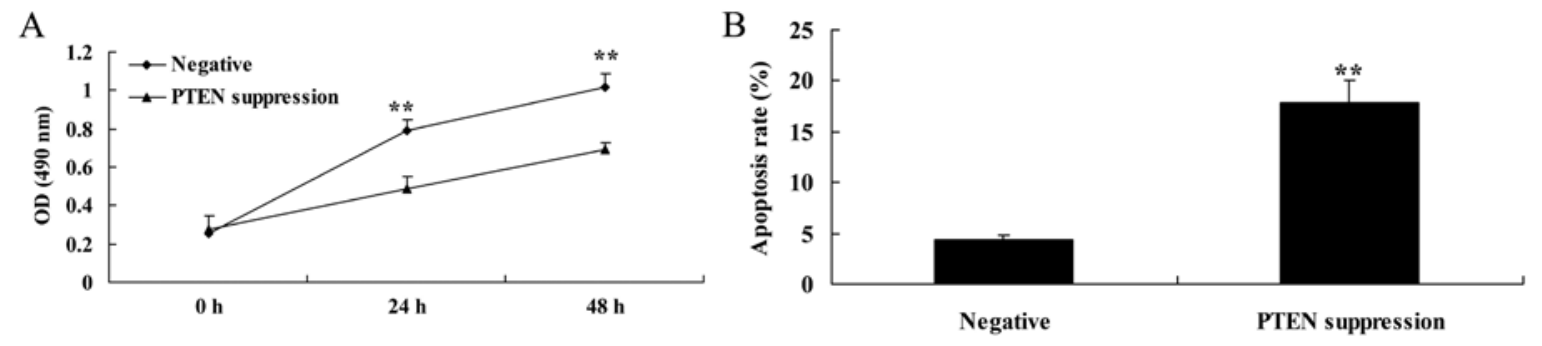

Figure 3. Suppression of PTEN decreases cell proliferation and induces the apoptosis of MCF-7 cells. (A) Suppression of PTEN decreased the cell proliferation and (B) induced apoptosis in the MCF-7 cells; ${ }^{* *} \mathrm{p}<0.01$ vs. the negative group.

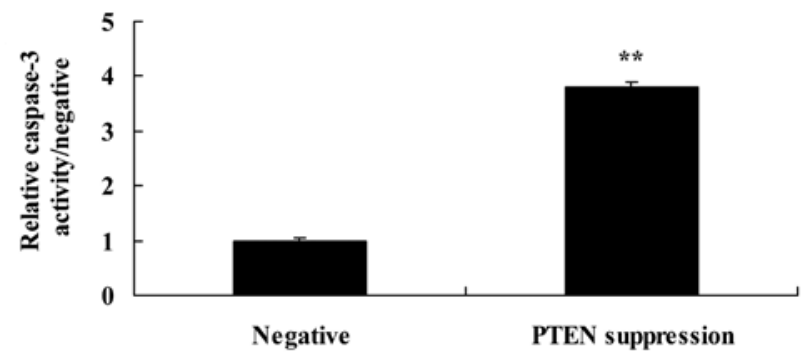

Figure 4. Suppression of PTEN increased the caspase-3 activity in the MCF-7 cells; ${ }^{* *} \mathrm{p}<0.01$ vs. the negative group.

than these levels in the para-carcinoma tissue (Fig. 2A and B). TOP2A protein and miRNA expression in breast cancer tissue were observably higher than these levels in the para-carcinoma tissues (Fig. 2C and D).

Suppression of PTEN decreases cell proliferation and induces apoptosis in MCF-7 cells. In order to determine whether PTEN is involved in the regulation of MCF-7 cell death, a PTEN inhibitor was used to suppress the expression of PTEN protein. As shown in Fig. 3A, the PTEN inhibitor significantly decreased cell viability of the MCF-7 cells in a time-dependent manner, compared with the negative group. As shown in
Fig. 3B, PTEN inhibitor also significantly induced apoptosis in the MCF-7 cells, compared with that noted in the negative group.

Suppression of PTEN increases caspase-3 activity and Bax protein expression in MCF-7 cells. We demonstrated that suppression of PTEN increased caspase-3 activity and Bax protein expression in the MCF-7 cells. As shown in Figs. 4, and 5A and B, suppression of PTEN significantly increased caspase- 3 activity and Bax protein expression in the MCF-7 cells.

Suppression of PTEN reduces the TUBB3 protein expression in MCF-7 cells. To understand the mechanism of PTEN underlying MCF-7 cell death, TUBB3 protein expression was measured using western blot analysis. As shown in Fig. 5B and C, the suppression of PTEN significantly reduced the protein expression of TUBB3 in the MCF-7 cells, compared with that noted in the negative group.

Suppression of PTEN reduces the TOP $2 A$ protein expression in MCF-7 cells. To understand the mechanism of PTEN underlying MCF-7 cell death, TOP2A protein expression was also measured using western blot analysis. As indicated in Fig. 5B and D, the suppression of PTEN significantly reduced 
A

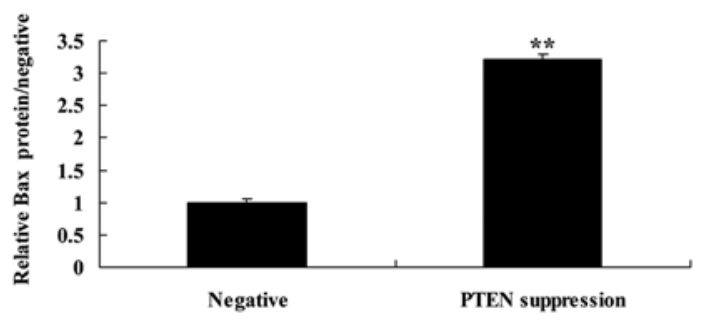

$\mathrm{C}$

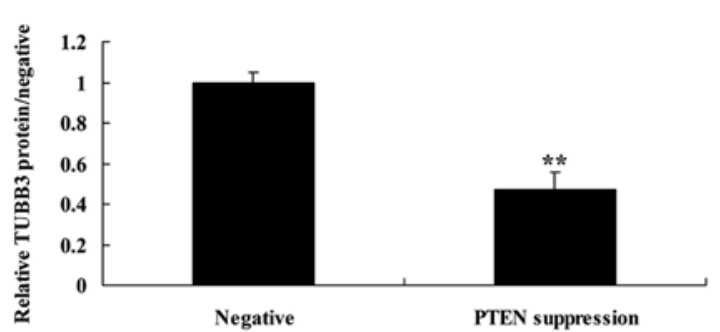

B

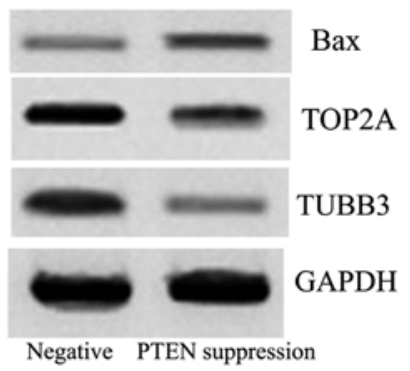

$\mathrm{D}$

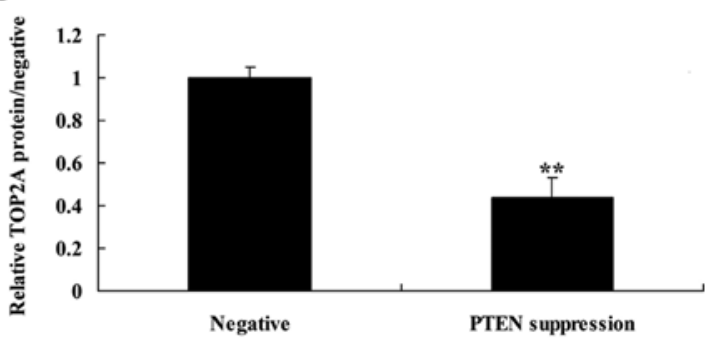

Figure 5. Suppression of PTEN affects TUBB3, TOP2A and Bax protein expression in the MCF-7 cells. (A, C and D) Effects of the suppression of PTEN on TUBB3, TOP2A and Bax protein expression as determined using statistical analysis. (B) Western blot analysis of TUBB3, TOP2A and Bax protein expression in MCF-7 cells; ${ }^{* *} \mathrm{p}<0.01$ vs. the negative group.

A

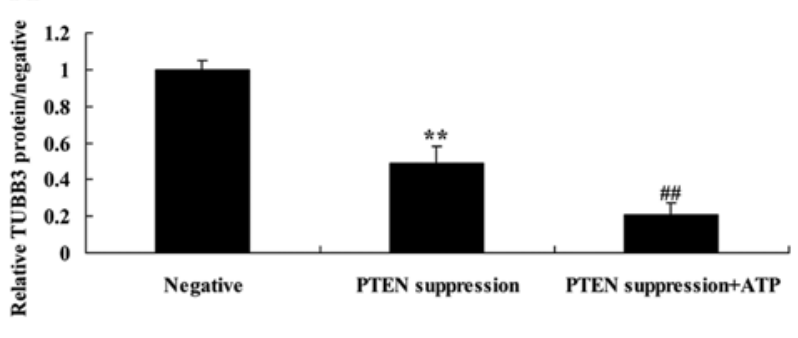

$\mathrm{C}$

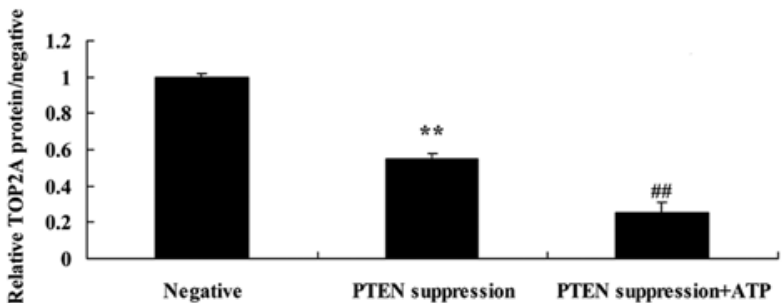

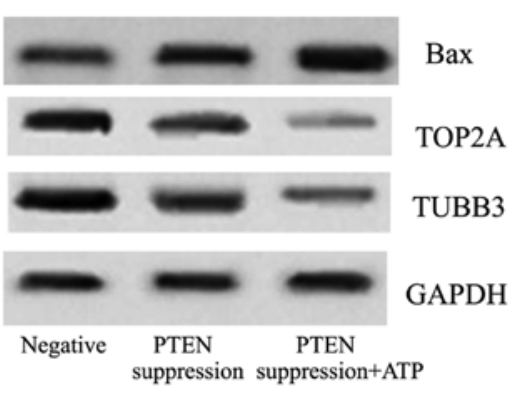

D

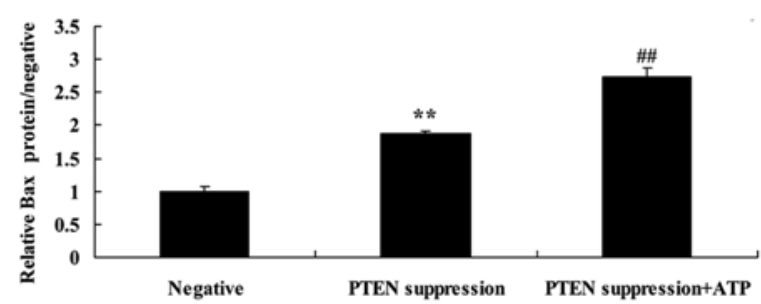

Figure 6. Treatment with ATP affects TUBB3, TOP2A and Bax protein expression in MCF-7 cells following suppression of PTEN. (A, C and D) Treatment of ATP affects TUBB3, TOP2A and Bax protein expression as determined using statistical analysis. (B) Western blot analysis of TUBB3, TOP2A and Bax protein expression in MCF-7 cells following suppression of PTEN and/or ATP; ${ }^{* *} \mathrm{p}<0.01$ vs. the negative group; ${ }^{\# \#} \mathrm{p}<0.01$ vs. the PTEN suppression group.

the protein expression of TOP2A in the MCF-7 cells, compared with that noted in the negative group.

ATP inhibits TUBB3 protein expression in MCF-7 cells following suppression of PTEN. To ascertain whether ATP regulates the effect of PTEN on TUBB3 expression in MCF-7 cells, ATP $(5 \mu \mathrm{M})$ was added to the MCF-7 cells following suppression of PTEN. Particularly, ATP inhibited TUBB3 expression in the MCF-7 cells following suppression of PTEN, compared with MCF-7 cells following suppression only of PTEN (Fig. 6A and B).

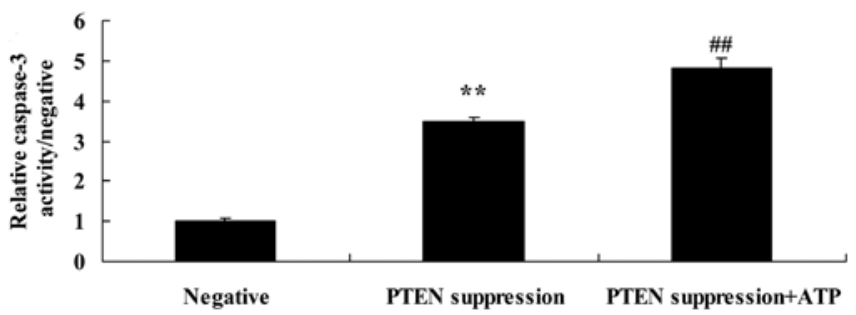

Figure 7. Effects of the suppression of PTEN and/or ATP on caspase-3 activity in MCF-7 cells; ${ }^{* *} \mathrm{p}<0.01$ vs. the negative group; ${ }^{\# \#} \mathrm{p}<0.01 \mathrm{vs}$. the PTEN suppression group. 

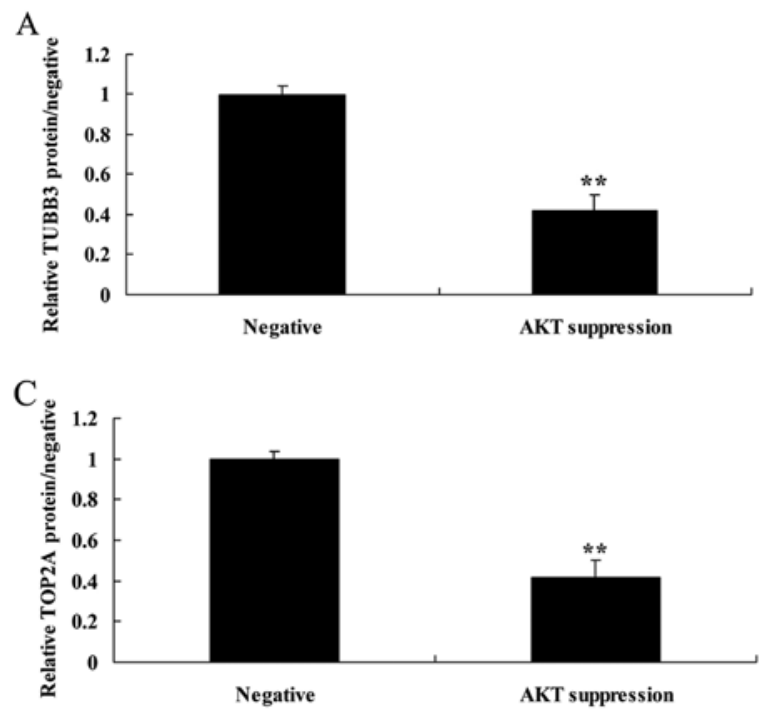

B

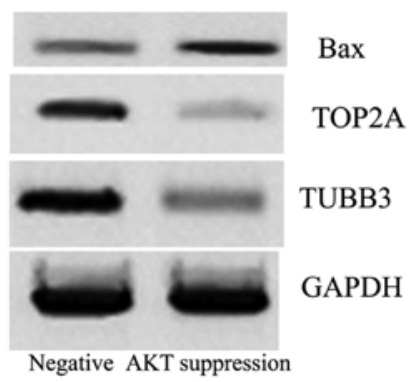

$\mathrm{D}$

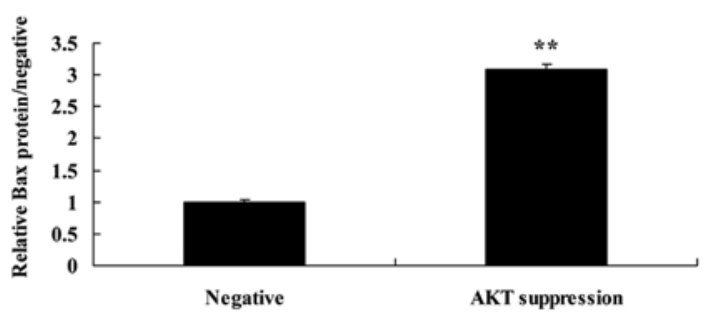

Figure 8. Suppression of p-AKT affects TUBB3, TOP2A and Bax protein expression in MCF-7 cells. (A, C and D) Effects of the suppression of p-AKT on TUBB3, TOP2A and Bax protein expression as determined using statistical analysis. (B) Western blot analysis of TUBB3, TOP2A and Bax protein expression in the MCF-7 cells; ${ }^{* *} \mathrm{p}<0.01$ vs. the negative group.
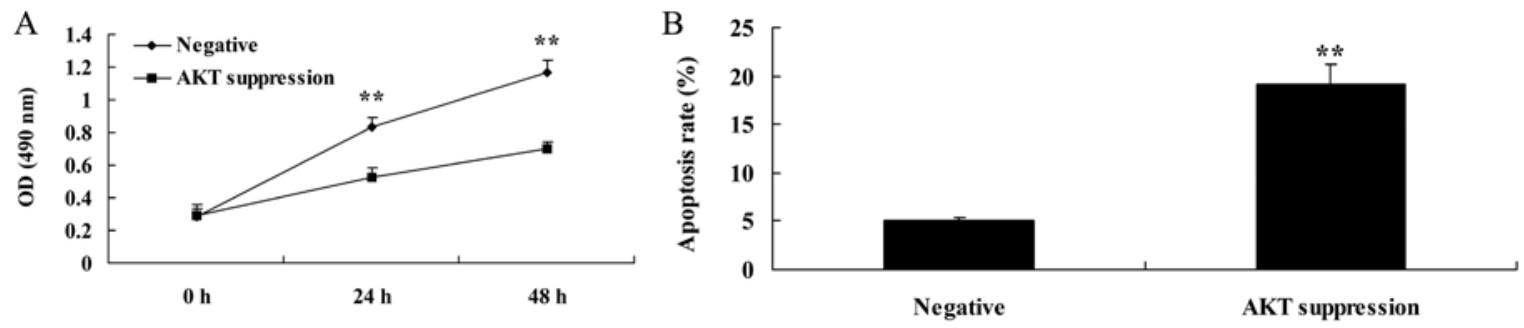

Figure 9. Suppression of p-AKT affects cell proliferation and induction of apoptosis in MCF-7 cells. (A) Suppression of p-AKT decreased cell proliferation and (B) induced apoptosis in the MCF-7 cells; ${ }^{* *} \mathrm{p}<0.01$ vs. the negative group.

ATP reduces TOP $2 A$ protein expression in MCF-7 cells following suppression of PTEN. To ascertain whether ATP regulates the effect of PTEN on TOP2A expression in MCF-7 cells, MCF-7 cells were treated with ATP $(5 \mu \mathrm{M})$ following suppression of PTEN. Analogously, increased ATP suppressed TOP2A protein expression in the MCF-7 cells following suppression of PTEN, compared with MCF-7 cells following suppression only of PTEN (Fig. 6B and C).

Treatment of ATP further increases caspase-3 activity and Bax protein expression in MCF-7 cells following suppression of PTEN. To ascertain whether ATP regulates the effect of PTEN on MCF-7 cell apoptosis, following suppression of PTEN, ATP was added to the MCF-7 cells. We found that treatment with ATP increased caspase-3 activity and Bax protein expression in the MCF-7 cells following suppression of PTEN, compared with MCF-7 cells following suppression only of PTEN (Figs. 6B and D, and 7).

Suppression of $p$-AKT reduces TUBB3 protein expression in MCF-7 cells. To further understand the mechanism of p-AKT in MCF-7 cell death, TUBB3 protein expression was selected and measured using western blot analysis. As shown in Fig. 8A and B, the suppression of p-AKT significantly

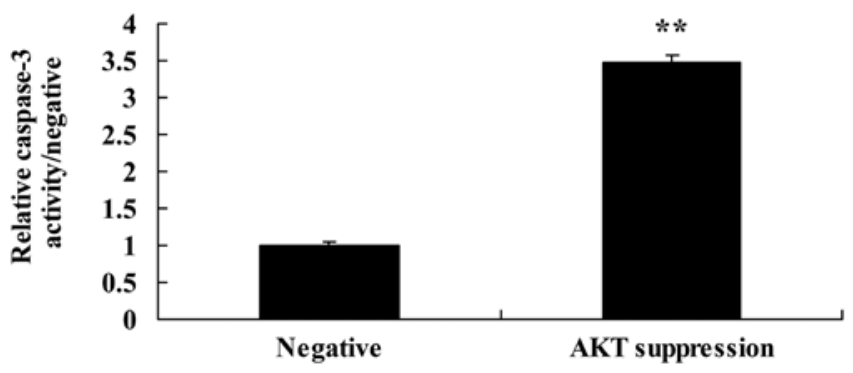

Figure 10. Suppression of p-AKT increased caspase-3 activity in the MCF-7 cells; ${ }^{* *} \mathrm{p}<0.01 \mathrm{vs}$. the negative group.

suppressed the protein expression of TUBB3 in the MCF-7 cells, compared with the level in the negative group.

Suppression of $p$-AKT reduces TOP $2 A$ protein expression in MCF-7 cells. To further understand the mechanism of p-AKT in MCF-7 cell death, TOP2A protein expression was also selected and measured using western blot analysis. As indicated in Fig. 8B and C, the protein expression of TOP2A in MCF-7 cells was significantly reduced following the suppression of $\mathrm{p}-\mathrm{AKT}$, as compared with the level in the negative group. 
A

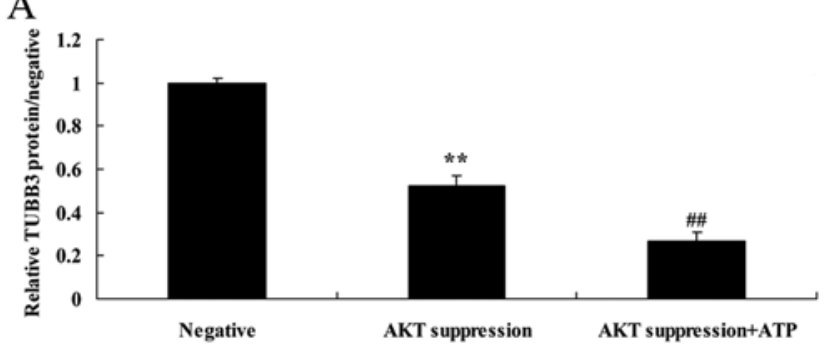

$\mathrm{C}$

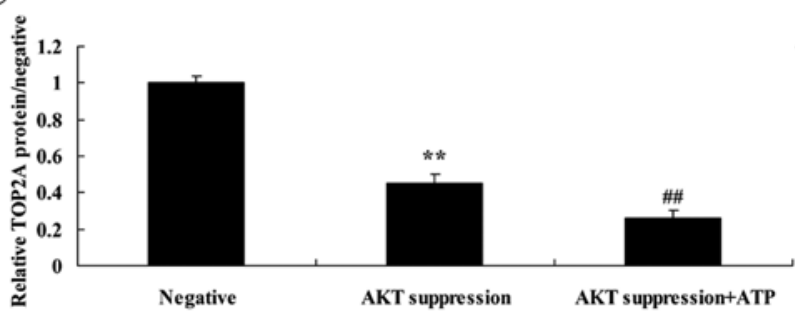

$\mathrm{B}$

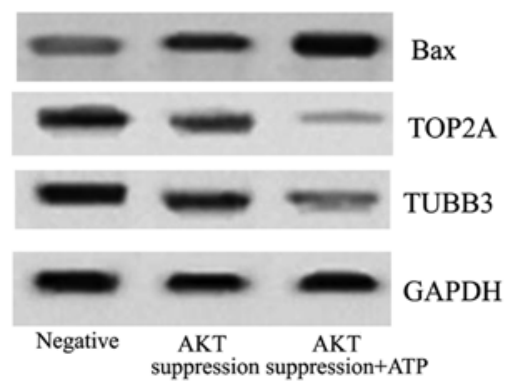

$\mathrm{D}$

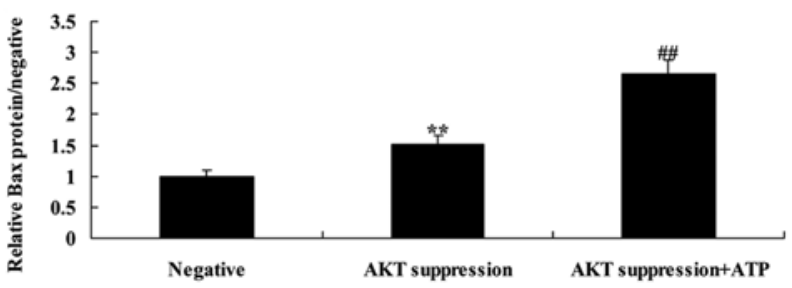

Figure 11. Treatment with ATP affects TUBB3, TOP2A and Bax protein expression in MCF-7 cells following suppression of p-AKT. (A, C and D) Treatment with ATP affects TUBB3, TOP2A and Bax protein expression as determined using statistical analysis. (B) Western blot analysis of TUBB3, TOP2A and Bax protein expression in MCF-7 cells following suppression of $\mathrm{p}-\mathrm{AKT}$ and/or ATP; ${ }^{* *} \mathrm{p}<0.01$ vs. the negative group; ${ }^{\# \#} \mathrm{p}<0.01$ vs. the AKT suppression group.

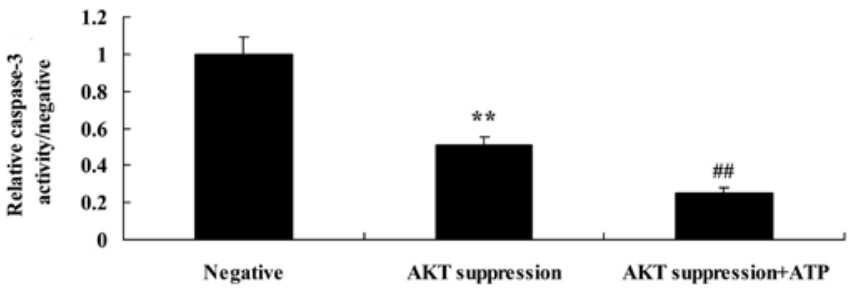

Figure 12. Treatment of ATP decreases caspase-3 activity in MCF-7 cells following suppression of $\mathrm{p}-\mathrm{AKT} ;{ }^{* *} \mathrm{p}<0.01$ vs. the negative group; ${ }^{\# \#} \mathrm{p}<0.01$ vs. the AKT suppression group.

Suppression of $p$-AKT reduces cell proliferation and induced apoptosis in MCF-7 cells. In order to further determine whether AKT is involved in the regulation of MCF-7 cell death, p-AKT inhibitor was used to suppress the expression of PTEN protein. As shown in Fig. 9A, the p-AKT inhibitor significantly decreased the cell viability of the MCF-7 cells in a time-dependent manner, compared with that noted in the negative group. As shown in Fig. 9B, MCF-7 cell apoptosis was also induced by the p-AKT inhibitor, compared with the rate noted in the negative group.

Suppression of p-AKT increases caspase-3 activity and Bax protein expression in MCF-7 cells. We also demonstrated the effect of the suppression of p-AKT on caspase- 3 activity and Bax protein expression in MCF-7 cells. As shown in Figs. $8 \mathrm{~B}$ and $\mathrm{D}$, and 10 , the suppression of p-AKT significantly increased Bax protein expression and caspase- 3 activity in the MCF-7 cells, compared with these levels in the negative group.

Treatment with ATP inhibits TUBB3 protein expression in MCF-7 cells following suppression of $p$-AKT. To further ascertain whether ATP regulates the effect of p-AKT on TUBB3 expression in MCF-7 cells, ATP $(5 \mu \mathrm{M})$ was added to the MCF-7 cells following suppression of p-AKT. Specifically, ATP significantly inhibited TUBB3 expression in the MCF-7 cells following suppression of $\mathrm{p}$-AKT, compared with that noted in the MCF-7 cells following suppression only of p-AKT (Fig. 11A and B).

Treatment of ATP suppresses TOP $2 A$ protein expression in $M C F-7$ cells following suppression of $p-A K T$. To ascertain whether ATP regulates the effect of p-AKT on TOP2A expression in MCF-7 cells, ATP $(5 \mu \mathrm{M})$ was added to the MCF-7 cells following suppression of p-AKT. Analogously, treatment with ATP suppressed TOP2A protein expression in the MCF-7 cells following suppression of p-AKT, compared with that noted in the MCF-7 cells following suppression only of p-AKT (Fig. 11B and C).

Treatment with ATP decreases caspase-3 activity and increases Bax protein expression in MCF-7 cells following suppression of $p-A K T$. To ascertain whether ATP regulates the effect of PTEN on MCF-7 cell apoptosis following suppression of PTEN, ATP was added to the MCF-7 cells. We found that treatment with ATP decreased caspase-3 activity in the MCF-7 cells following suppression of p-AKT, compared with that in the MCF-7 cells following suppression only of p-AKT (Fig. 12). Meanwhile, treatment with ATP decreased Bax protein expression in the MCF-7 cells following suppression of p-AKT, compared with that noted in the MCF-7 cells following suppression only of p-AKT (Fig. 11B and D).

\section{Discussion}

Breast cancer has become a serious disease that endangers the health of all women as well as Chinese women, particularly those in the cities of China (4). During the past 20 years, along with the development of chemotherapy, hormonal and targeted therapies, the treatment of breast cancer has 
significantly improved (15). Cytotoxic chemotherapeutic drugs with anthracycline as representative have been widely used in breast cancer; however, in recent years, there are few studies concerning biomarkers related to the prediction of the outcome of chemotherapy (16).

Multiple studies have shown that TUBB3 is not only expressed in normal tissues, but also in cancer tissues (such as non-small cell lung cancer). However, the amount in adjacent normal tissues is far less than that in cancerous tissues (17). TUBB3 is mainly expressed in the nucleus, and immunohistochemistry showing brown granules indicates positive expression (18). Research has confirmed the correlation between TUBB3 expression and the chemosensitivity of taxanes, and TUBB3 has been confirmed as a prognostic predictor of various solid tumors such as breast, ovarian, prostate and lung cancer (6). In the present study, the DFS and OS of human breast cancer patients with TUBB3- or TOP2A-positive tumors were lower than the DFS and OR of the patients with TUBB3- or TOP2A-negative tumors.

During the past 10 years, a large number of research results suggest that HER2-positive breast cancer may be resistant to CMF and TAM regimens. Compared with the non-anthracycline drugs, HER2-positive breast cancer is more sensitive to the treatment of anthracycline-containing drugs. However, recent clinical studies have shown that, in fact, the predictive value of the efficacy of anthracycline drugs on HER2-positive breast cancer may be affectd by the synergistic amplification of the TOP2A gene (19). The TOP2A gene is adjacent to the HER2 gene, located in the 21 site of the long arm of chromosome 17, which is expressed as amplification or deletion in breast cancer with HER2 gene amplification. TOP2A encoded protein-topoisomerase is a key protease involved in DNA repair, cell cycle regulation and chromosome division (12). Topoisomerase 11 is also a target of anthracycline drugs, such as adriamycin or epirubicin. Therefore, TOP2A is considered to be a more valuable marker than HER2 for the predication of anthracycline efficacy. More importantly, we found that the suppression of PTEN reduced cell proliferation and induced apoptosis and caspase- 3 activity, suppressed the protein expression of TUBB3 and TOP2A in MCF-7 cells through regulation of ATP.

An abnormality in the HER2 signaling pathway is an important mechanism for the development of breast cancer, in which the key gene is a predictor for the targeted drug efficacy of breast cancer (20). Patients with HER2 overexpression can achieve significant efficacy using trastuzumab; however, patients with loss of PTEN or PI3K/AKT mutations show resistance to trastuzumab. The abnormality of the key genes mentioned above leads to the activation of downstream signaling of HER2. Although trastuzumab has adequate targets, it cannot control the abnormal changes in the downstream signal, and cannot achieve tumor cell proliferation. With the widespread use of anthracyclines and the gradual increase in patient resistance, it was found through clinical detection that breast cancer patients with HER2 overexpression show anthracycline sensitivity, and as TOP2A and HER2 genes are located on chromosome 17 , and very close, there may be mutual regulation in the coding region (12). Research has demonstrated that HER2 overexpression is also a significant predictor for anthracycline efficacy (11).
The PTEN/PI3K/Akt signaling pathway exists in a wide variety of tumor cells, and pathway activation can inhibit apoptosis to promote cell cycle progression, thereby promoting cell growth and proliferation. It is also involved in tumor angiogenesis, which plays an important role in tumor formation, and in tumor invasion and metastasis (21). PTEN can inhibit tumor formation by the negative regulation of signaling pathways, and inactivation or the mutation of the PTEN gene can reduce or lose the inhibitory effect on the pathway to cause cancer cell growth (22). In the PI3K/AKT signal transduction process, when PI3K is activated, PIP3 as a second messenger can activate numerous downstream signaling molecules, thereby resulting in further signal transduction. The PI3K signaling pathway plays an important role in many pathophysiological processes such as cell differentiation, apoptosis, proliferation, migration, vesicular transport, angiogenesis and cell malignant transformation (23). Our results also suggest that the suppression of AKT reduced cell proliferation and induced apoptosis and caspase- 3 activity, and suppressed the protein expression of TUBB3 and TOP2A in MCF-7 cells through regulation of ATP.

In conclusion, the present study also demonstrated that PTEN/AKT signaling regulates the expression of TUBB3 and TOP2A and affects the cell growth and apoptosis of human breast cancer MCF-7 cells through ATP and caspase-3 signaling pathway. Therefore, this report suggests a potential predictive role of TUBB3 and TOP2A in therapeutic outcome of human breast cancer to avoid the use of ineffective therapies.

\section{Acknowledgements}

The present study was supported by the Guangdong Province Science and Technology Plan (2014A020212498).

\section{References}

1. Forbes JF, Sestak I, Howell A, Bonanni B, Bundred N, Levy C, von Minckwitz G, Eiermann W, Neven P, Stierer M, et al; IBIS-II investigators: Anastrozole versus tamoxifen for the prevention of locoregional and contralateral breast cancer in postmenopausal women with locally excised ductal carcinoma in situ (IBIS-II DCIS): A double-blind, randomised controlled trial. Lancet 387: 866-873, 2016.

2. Pu Z, Zhang X, Chen Q, Yuan X and Xie H: Establishment of an expression platform of OATP1B1 388GG and 521CC genetic polymorphism and the therapeutic effect of tamoxifen in MCF-7 cells. Oncol Rep 33: 2420-2428, 2015.

3. Aihara T, Yokota I, Hozumi Y, Aogi K, Iwata H, Tamura M, Fukuuchi A, Makino H, Kim R, Andoh M, et al: Anastrozole versus tamoxifen as adjuvant therapy for Japanese postmenopausal patients with hormone-responsive breast cancer: Efficacy results of long-term follow-up data from the N-SAS $\mathrm{BC} 0_{3}$ trial. Breast Cancer Res Treat 148: 337-343, 2014.

4. Wang J, Xu B, Yuan P, Ma F, Li Q, Zhang P, Cai R, Fan Y, Luo Y and Li Q: Capecitabine combined with docetaxel versus vinorelbine followed by capecitabine maintenance medication for first-line treatment of patients with advanced breast cancer: phase 3 randomized trial. Cancer 121: 3412-3421, 2015.

5. Witt CM, Außerer O, Baier S, Heidegger H, Icke K, Mayr O, Mitterer M, Roll S, Spizzo G, Scherer A, et al: Effectiveness of an additional individualized multi-component complementary medicine treatment on health-related quality of life in breast cancer patients: A pragmatic randomized trial. Breast Cancer Res Treat 149: 449-460, 2015.

6. Xu YC, Zhang FC, Li JJ, Dai JQ, Liu Q, Tang L, Ma Y, Xu Q, Lin XL, Fan HB, et al: RRM1, TUBB3, TOP2A, CYP19A1, CYP2D6: Difference between mRNA and protein expression in predicting prognosis of breast cancer patients. Oncol Rep 34: 1883-1894, 2015. 
7. Leng XF, Chen MW, Xian L, Dai L, Ma GY and Li MH: Combined analysis of mRNA expression of ERCC1, BAG-1, BRCA1, RRM1 and TUBB3 to predict prognosis in patients with non-small cell lung cancer who received adjuvant chemotherapy. J Exp Clin Cancer Res 31: 25, 2012.

8. Huang ZL, Cao X, Luo RZ, Chen YF, Zhu LC and Wen Z: Analysis of ERCC1, BRCA1, RRM1 and TUBB3 as predictors of prognosis in patients with non-small cell lung cancer who received cisplatin-based adjuvant chemotherapy: A prospective study. Oncol Lett 11: 299-305, 2016.

9. Narvi E, Jaakkola K, Winsel S, Oetken-Lindholm C, Halonen P, Kallio L and Kallio MJ: Altered TUBB3 expression contributes to the epothilone response of mitotic cells. Br J Cancer 108: 82-90, 2013.

10. Huijsmans CJ, van den Brule AJ, Rigter H, Poodt J, van der Linden JC, Savelkoul PH, Hilbink M and Hermans MH Allelic imbalance at the $H E R 2 / T O P 2 A$ locus in breast cancer. Diagn Pathol 10: 56, 2015.

11. Fasching PA, Weihbrecht S, Haeberle L, Gasparyan A, Villalobos IE, Ma Y, Ekici AB, Wachter DL, Hartmann A, Beckmann MW, et al: HER2 and TOP2A amplification in a hospital-based cohort of breast cancer patients: Associations with patient and tumor characteristics. Breast Cancer Res Treat 145: 193-203, 2014

12. Fountzilas G, Valavanis C, Kotoula V, Eleftheraki AG, Kalogeras KT, Tzaida O, Batistatou A, Kronenwett R, Wirtz RM, Bobos M, et al: HER2 and TOP2A in high-risk early breast cancer patients treated with adjuvant epirubicin-based dose-dense sequential chemotherapy. J Transl Med 10: 10, 2012.

13. Li SZ, Qiao SF, Zhang JH and Li K: Quercetin increase the chemosensitivity of breast cancer cells to doxorubicin via PTEN/Akt pathway. Anticancer Agents Med Chem 15: 1185-1189, 2015.

14. Su R, Nan H, Guo H, Ruan Z, Jiang L, Song Y and Nan K: Associations of components of PTEN/AKT/mTOR pathway with cancer stem cell markers and prognostic value of these biomarkers in hepatocellular carcinoma. Hepatol Res: Mar 2, 2016 (Epub ahead of print). doi: 10.1111/hepr.12687.

15. Zheng S, Song QK, Zhao L, Huang R, Sun L, Li J, Fan JH, Zhang BN, Yang HJ, Xu F, et al: Characteristics of mammary Paget's disease in China: A national-wide multicenter retrospective study during 1999-2008. Asian Pac J Cancer Prev 13: $1887-1893,2012$.
16. Seidman AD, Chan S, Wang J, Zhu C, Xu C and Xu B: A pooled analysis of gemcitabine plus docetaxel versus capecitabine plus docetaxel in metastatic breast cancer. Oncologist 19: 443-452, 2014.

17. Sun S, Shi W, Wu Z, Zhang G, Yang BO and Jiao S: Prognostic significance of the mRNA expression of ERCCl, RRM1, TUBB3 and TYMS genes in patients with non-small cell lung cancer. Exp Ther Med 10: 937-941, 2015.

18. Zou ZQ, Du YY, Sui G and Xu SN: Expression of TS, RRM1, ERCC1, TUBB3 and STMN1 genes in tissues of non-small cell lung cancer and its significance in guiding postoperative adjuvant chemotherapy. Asian Pac J Cancer Prev 16: 3189-3194, 2015.

19. Nakagawa M, Bando Y, Nagao T, Takai C, Ohnishi T, Honda J, Moriya T, Izumi K, Takahashi M, Tangoku A, et al: Among triple-negative breast cancers, HER2(0) breast cancer shows a strong tendency to be basal-like compared with HER2(1+) breast cancer: preliminary results. Breast Cancer 19: 54-59, 2012.

20. Yang F, Lyu S, Dong S, Liu Y, Zhang X and Wang O: Expression profile analysis of long noncoding RNA in HER-2-enriched subtype breast cancer by next-generation sequencing and bioinformatics. Onco Targets Ther 9: 761-772, 2016

21. Fu X, Tian J, Zhang L, Chen Y and Hao Q: Involvement of microRNA-93, a new regulator of PTEN/Akt signaling pathway, in regulation of chemotherapeutic drug cisplatin chemosensitivity in ovarian cancer cells. FEBS Lett 586: 1279-1286, 2012.

22. Shih MC, Chen JY, Wu YC, Jan YH, Yang BM, Lu PJ, Cheng HC, Huang MS, Yang CJ, Hsiao M, et al: TOPK/PBK promotes cell migration via modulation of the PI3K/PTEN/AKT pathway and is associated with poor prognosis in lung cancer. Oncogene 31: 2389-2400, 2012.

23. Qadir XV, Han C, Lu D, Zhang J and Wu T: miR-185 inhibits hepatocellular carcinoma growth by targeting the DNMT1/PTEN/Akt pathway. Am J Pathol 184: 2355-2364, 2014. 